Many worlds and modality in the interpretation of quantum mechanics: An algebraic approach

G. Domenech' , H. Freytes', and C. de Ronde

Citation: Journal of Mathematical Physics 50, 072108 (2009); doi: 10.1063/1.3177454

View online: http://dx.doi.org/10.1063/1.3177454

View Table of Contents: http://aip.scitation.org/toc/jmp/50/7

Published by the American Institute of Physics

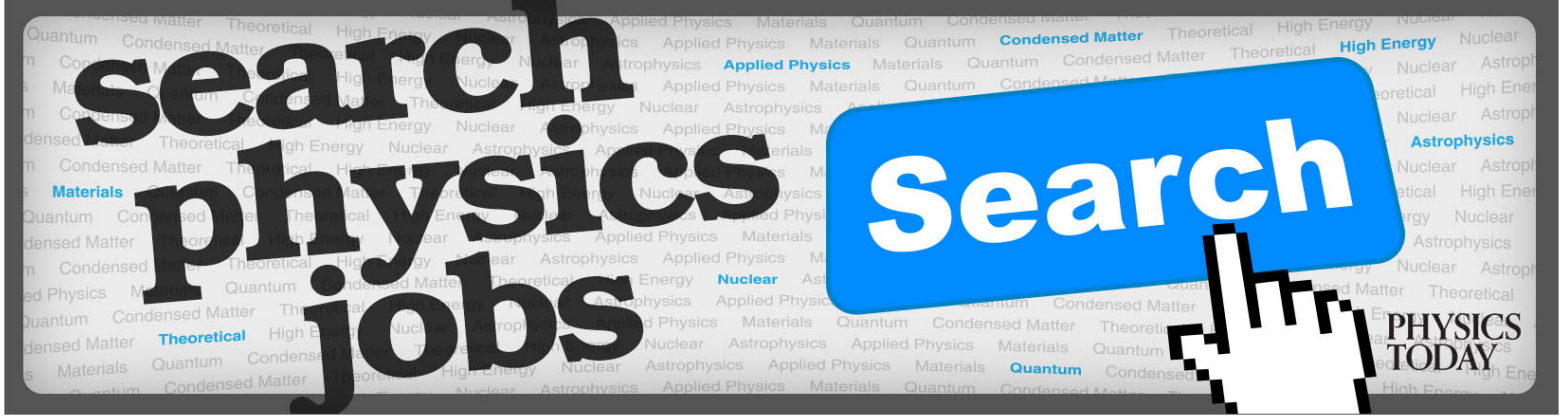




\title{
Many worlds and modality in the interpretation of quantum mechanics: An algebraic approach
}

\author{
G. Domenech, ${ }^{1,3, a)}$ H. Freytes, ${ }^{2, b)}$ and C. de Ronde ${ }^{3,4}$ \\ ${ }^{1}$ Instituto de Astronomía y Física del Espacio (IAFE), Casilla de Correo 67, Sucursal 28, \\ 1428 Buenos Aires, Argentina \\ ${ }^{2}$ Universita degli Studi di Cagliari, Via Is Mirrionis 1, 09123 Cagliari, Italy \\ ${ }^{3}$ Center Leo Apostel (CLEA), Vrije Universiteit Brussel, Krijgskunderstraat 33, 13-1160 \\ Brussels, Belgium \\ ${ }^{4}$ Foundations of the Exact Sciences (FUND), Brussels Free University, Krijgskundestraat \\ 33, 1160 Brussels, Belgium
}

(Received 26 August 2008; accepted 25 June 2009; published online 31 July 2009)

\begin{abstract}
Many world interpretations (MWIs) of quantum mechanics avoid the measurement problem by considering every term in the quantum superposition as actual. A seemingly opposed solution is proposed by modal interpretations (MIs) which state that quantum mechanics does not provide an account of what "actually is the case," but rather deals with what "might be the case," i.e., with possibilities. In this paper we provide an algebraic framework which allows us to analyze in depth the modal aspects of MWI. Within our general formal scheme we also provide a formal comparison between MWI and MI, in particular, we provide a formal understanding of why-even though both interpretations share the same formal structure-MI fall pray of Kochen-Specker-type contradictions while MWI escape them. () 2009 American Institute of Physics. [DOI: 10.1063/1.3177454]
\end{abstract}

\section{INTRODUCTION: MANY WORLDS AND MODALITY}

Today, almost 50 years after its birth in 1957, the many world interpretation (MWI) of quantum mechanics still is one important line of investigation within the many interpretations of quantum theory. MWI is considered to be a direct conclusion from Everett's ${ }^{11}$ first proposal in terms of "relative states." Everett's idea was to let quantum mechanics find its own interpretation, making justice to the symmetries inherent in the Hilbert space formalism in a simple and convincing way. ${ }^{7}$ In this paper we will not address the main argumentative lines of discussion raised for and against MWI (see, for example, Refs. 4, 6, and 20). Rather, we shall concentrate in its relation to the formal structure of quantum mechanics and provide an algebraic frame which will allow us to discuss the notion of logical possibility within it.

The main idea behind MWI is that superpositions refer to collections of worlds, in each of which exactly one value of an observable, which corresponds to one of the terms in the superposition, is realized. Apart from being simple, the claim is that it possesses a natural fit to the formalism, respecting its symmetries. This provides a solution to the measurement problem by assuming that each one of the terms in the superposition is actual in its own correspondent world. Thus, it is not only the single value which we see in "our world" which gets actualized but rather, that a branching of worlds takes place in every measurement, giving rise to a multiplicity of worlds with their corresponding actual values. The possible splits of the worlds are determined by the laws of quantum mechanics.

Another proposed solution to the so-called measurement problem has been developed in the frame of modal interpretations (MIs). ${ }^{5,18,21}$ According to these interpretations "the quantum for-

\footnotetext{
${ }^{a)}$ Electronic mail: domenech@iafe.uba.ar.

b) Permanent address, Instituto Argentino de Matemática IAM-CONICET.
} 
malism does not tell us what actually is the case in the physical world, but rather provides us with a list of possibilities and their probabilities. The modal viewpoint is therefore that quantum theory is about what may be the case, in philosophical jargon, quantum theory is about modalities." Instead of actualizing every term in the superposition, MI claims that each term remains possible, evolving with the Schrödinger equation of motion.

Although MWI and MI share the same formal orthodox scheme, there are but few comparisons in the literature. ${ }^{4,12}$ In this paper we develop an algebraic framework which allows us to analyze and discuss the modal aspects of MWI. Within this new formal account, we can also provide a rigorous comparison between MWI and MI. In particular, we can give a formal understanding of why MI fall pray of Kochen-Specker (KS)-type contradictions ${ }^{1,17}$ while MWI escapes them.

In Sec. II, we introduce basic notions about lattice theory that will be necessary later. In Sec. III, we provide a general discussion on contextuality and modality in quantum mechanics. In Sec. IV, we develop a new algebraic frame for MWI. In Sec. V, we formally compare MWI to MI.

\section{BASIC NOTIONS}

We freely use all basic notions of universal algebra that can be found in Ref. 2. Let $\sigma$ be a type of algebras and let $\mathcal{A}$ be a class of algebras of type $\sigma$. We denote by $\operatorname{Term}_{\mathcal{A}}$ the absolutely free algebra of type $\sigma$ built from the set of variables $X=\left\{x_{1}, x_{2}, \ldots\right\}$. Let $A \in \mathcal{A}$. If $t \in \operatorname{Term}_{\mathcal{A}}$ and $a_{1}, \ldots, a_{n} \in A$, by $t^{A}\left[a_{1}, \ldots, a_{n}\right]$ we denote the result of the application of the term operation $t^{A}$ to the elements $a_{1}, \ldots, a_{n} \in A$. If $B \subset A$, the subalgebra of $A$ generated by $B$ is denoted by $\langle B\rangle_{A}$.

Now we recall from Refs. 16 and 19 some notions of universal algebra and lattice theory that will play an important role in what follows. Let $\mathcal{L}$ be a lattice and $a, b \in \mathcal{L}$. We say that $b$ covers $a$ if and only if $a<b$, and moreover there exists no $x \in \mathcal{L}$, such that $a<x<b$ for any $x$. Suppose that $\mathcal{L}$ is a bounded lattice with 0 the minimum element and 1 the maximum element. An element $p \in \mathcal{L}$ is called an atom if and only $p$ covers 0 and a coatom if and only if 1 covers $p$. $\mathcal{L}$ is said to be an atomistic lattice if and only if for each $x \in \mathcal{L}-\{0\}, x=\vee\{p \leq x: p$ is an atom $\}$. An element $c \in \mathcal{L}$ is said to be a complement of $a$ if and only if $a \wedge c=0$ and $a \vee c=1$. Let $\mathcal{L}=\langle\mathcal{L}, \vee, \wedge, 0,1\rangle$ be a bounded lattice. Given $a, b, c$ in $\mathcal{L}$, we write $(a, b, c) D$ if and only if $(a \vee b) \wedge c$ $=(a \wedge c) \vee(b \wedge c) ;(a, b, c) D^{*}$ if and only if $(a \wedge b) \vee c=(a \vee c) \wedge(b \vee c)$, and $(a, b, c) T$ if and only if $(a, b, c) D,(a, b, c) D^{*}$ hold for all permutations of $a, b, c$. An element $z$ of a lattice $\mathcal{L}$ is called central if and only if for all elements $a, b \in \mathcal{L}$ we have $(a, b, z) T$ and $z$ is complemented. We denote by $Z(\mathcal{L})$ the set of all central elements of $\mathcal{L}$ and it is called the center of $\mathcal{L}$.

A lattice with involution ${ }^{15}$ is an algebra $\langle\mathcal{L}, \vee, \wedge, \neg\rangle$ such that $\langle\mathcal{L}, \vee, \wedge\rangle$ is a lattice and $\neg$ is a unary operation on $\mathcal{L}$ that fulfills the following conditions: $\neg \neg x=x$ and $\neg(x \vee y)=\neg x \wedge \neg y$. An orthomodular lattice is an algebra $\langle\mathcal{L}, \wedge, \vee, \neg, 0,1\rangle$ of type $\langle 2,2,1,0,0\rangle$ that satisfies the following conditions.

(1) $\langle\mathcal{L}, \wedge, \vee, \neg, 0,1\rangle$ is a bounded lattice with involution.

(2) $x \wedge \neg x=0$.

(3) $x \vee(\neg x \wedge(x \vee y))=x \vee y$.

We denote by $\mathcal{O M L}$ the variety of orthomodular lattices. It is well known that if $\mathcal{H}$ is a Hilbert space then $\mathcal{L}(\mathcal{H})$, the lattice of closed subspaces of $\mathcal{H}$, also called Hilbert lattice, is an atomistic orthomodular lattice. Boolean algebras are orthomodular lattices satisfying the distributive law $x \wedge(y \vee z)=(x \wedge y) \vee(x \wedge z)$. We denote by 2 the Boolean algebra of two elements. If $\mathcal{L}$ is a bounded lattice then $Z(\mathcal{L})$ is a Boolean sublattice of $\mathcal{L}$ (Ref. 19, Theorem 4.15).

Let $A$ be a Boolean algebra. A subset $F$ of $A$ is called a filter if and only if it satisfies the following: if $a \in F$ and $a \leq x$ then $x \in F$ and if $a, b \in F$ then $a \wedge b \in F . F$ is a proper filter if and only if $F \neq A$ or, equivalently, $0 \notin F$. If $X \subseteq A$, the filter $F_{X}$ generated by $X$ is the minimum filter containing $X$. It is well known that $F_{X}=\left\{x \in A: \exists x_{1} \cdots x_{n} \in X\right.$ with $\left.x_{1} \wedge \cdots \wedge x_{n} \leq x\right\}$. Each filter $F$ in $A$ determines univocally a congruence in which the equivalence classes are given by $[x]=\{y$ $\in A: \neg x \vee y \in F$ and $x \vee \neg y \in F\}$. In this case the quotient set $A / \sim$, noted as $A / F$, is a Boolean 
algebra and the natural application $x \mapsto[x]$ is a Boolean homomorphism form $A$ to $A / F$. A proper filter $F$ is maximal if and only if the quotient algebra $A / F$ is isomorphic to 2 . It is well known that each proper filter can be extended to a maximal one. A very important property associated with maximal filters is the following: suppose that $x \not y$. Then there exists a maximal filter $F$ in $A$, such that $x \in F$ and $y \notin F$. We will refer to this result as the maximal filter theorem.

\section{CONTEXTUALITY AND MODALITY IN QUANTUM SYSTEMS}

In the usual terms of quantum logic, ${ }^{3,14}$ a property of a system is related to a subspace of the Hilbert space $\mathcal{H}$ of its (pure) states or, analogously, to the projector operator onto that subspace. A physical magnitude $\mathcal{M}$ is represented by an operator $\mathbf{M}$ acting over the state space. For bounded self-adjoint operators, conditions for the existence of the spectral decomposition $\mathbf{M}=\sum_{i} a_{i} \mathbf{P}_{i}$ $=\Sigma_{i} a_{i}\left|a_{i}\right\rangle\left\langle a_{i}\right|$ are satisfied. The real numbers $a_{i}$ are related to the outcomes of measurements of the magnitude $\mathcal{M}$ and projectors $\left|a_{i}\right\rangle\left\langle a_{i}\right|$ to the mentioned properties. Thus, the physical properties of the system are organized in the lattice of closed subspaces $\mathcal{L}(\mathcal{H})$. Moreover, each self-adjoint operator $\mathbf{M}$ has associated a Boolean sublattice $W_{\mathbf{M}}$ of $L(\mathcal{H})$ which we will refer to as the spectral algebra of the operator $\mathbf{M}$.

Assigning values to a physical quantity $\mathcal{M}$ is equivalent to establishing a Boolean homomorphism $v: W_{\mathbf{M}} \rightarrow \mathbf{2}$. Thus, we can say that it makes sense to use the "classical discourse"-this is, the classical logical laws are valid-within the context given by $\mathcal{M}$.

One may define a global valuation of the physical magnitudes over $\mathcal{L}(\mathcal{H})$ as a family of Boolean homomorphisms $\left(v_{i}: W_{i} \rightarrow \mathbf{2}\right)_{i \in I}$ such that $v_{i}\left|W_{i} \cap W_{j}=v_{j}\right| W_{i} \cap W_{j}$ for each $i, j \in I$, being $\left(W_{i}\right)_{i \in I}$ the family of Boolean sublattices of $\mathcal{L}(\mathcal{H}) .{ }^{2}$ This global valuation would give the values of all magnitudes at the same time maintaining a compatibility condition in the sense that whenever two magnitudes shear one or more projectors, the values assigned to those projectors are the same from every context. As we have proved in Ref. 8, the KS theorem in the algebraic terms of the previous definition rules out this possibility.

Theorem 3.1: If $\mathcal{H}$ is a Hilbert space such that $\operatorname{dim}(\mathcal{H})>2$, then a global valuation over $\mathcal{L}(\mathcal{H})$ is not possible.

This impossibility to assign values to the properties at the same time satisfying compatibility conditions is a weighty obstacle for the interpretation of the formalism.

We have introduced elsewhere ${ }^{9,10}$ a general modal scheme which extends the expressive power of the orthomodular structure to provide a rigorous framework for the Born rule and mainly, to discuss the restrictions posed by the KS theorem to possible properties. We recall here some notions that will be useful in our development.

First, we enriched the orthomodular structure with a modal operator taking into account the following considerations.

(1) Propositions about the properties of the physical system are interpreted in the orthomodular lattice of closed subspaces of $\mathcal{H}$. Thus, we retain this structure in our extension.

(2) Given a proposition about the system, it is possible to define a context from which one can predicate with certainty about it together with a set of propositions that are compatible with it and, at the same time, predicate probabilities about the other ones (Born rule). In other words, one may predicate truth or falsity of all possibilities at the same time, i.e., possibilities allow an interpretation in a Boolean algebra. In rigorous terms, for each proposition $p$, if we refer with $\diamond p$ to the possibility of $p$, then $\diamond p$ will be a central element of a orthomodular structure.

(3) If $p$ is a proposition about the system and $p$ occurs, then it is trivially possible that $p$ occurs. This is expressed as $p \leq \diamond p$.

(4) Let $p$ be a property appertaining to a context $\mathcal{M}$. Assuming that $p$ is an actual property (for example, the result of a filtering measurement) we may derive from it a set of propositions (perhaps not all of them encoded in the original Hilbert lattice of the system) which we call classical consequences. For example, let $q$ be another property of the system and assign to $q$ the probability $\operatorname{prob}(q)=r$ via the Born rule and taking into account the truth of $p$. Then equality $\operatorname{prob}(q)=r$ will be considered as a classical consequence of $p$. In fact, the main 
characteristic of this kind of classical consequences is that it is possible to simultaneously predicate the truth of all of them (and the falsity of their negations) whenever $p$ is true. The formal representation of the concept of classical consequence is the following: A proposition $t$ is a classical consequence of $p$ if and only if $t$ is in the center of an orthomodular lattice containing $p$ and satisfies the property $p \leq t$. These classical consequences are the same ones as those which would be obtained by considering the original actual property $p$ as a possible one $\diamond p$. Consequently $\diamond p$ must precede all classical consequences of $p$. This is interpreted in the following way: $\diamond p$ is the smallest central element greater than $p$.

From consideration 1, it follows that the original orthomodular structure is maintained. The other considerations are satisfied if we consider a modal operator $\diamond$ over an orthomodular lattice $\mathcal{L}$ defined as

$$
\diamond a=\operatorname{Min}\{z \in Z(\mathcal{L}): a \leq z\}
$$

with $Z(\mathcal{L})$ the center of $\mathcal{L}$. When this minimum exists for each $a \in \mathcal{L}$ we say that $\mathcal{L}$ is a Boolean saturated orthomodular lattice. On each Boolean saturated orthomodular lattice, we can define the necessity operator as a unary operation $\square$ given by $\square x=\neg \diamond \neg x$. We have shown that this enriched orthomodular structure can be axiomatized by equations conforming a variety denoted by $\mathcal{O} \mathcal{M} \mathcal{L}^{\diamond}{ }^{9}$ More precisely, each element of $\mathcal{O} \mathcal{M} \mathcal{L}^{\diamond}$ is an algebra $\langle\mathcal{L}, \wedge, \vee, \neg, \square, 0,1\rangle$ of type $\langle 2,2,1,1,0,0\rangle$ satisfying the following equations:

\section{S1 $\square x \leq x$,}

S2 $\square 1=1$,

S3 $\square \square x=\square x$,

S4 $\square(x \wedge y)=\square(x) \wedge \square(y)$,

S5 $y=(y \wedge \square x) \vee(y \wedge \neg \square x)$,

S6 $\square(x \vee \square y)=\square x \vee \square y$,

S7 $\square(\neg x \vee(y \wedge x)) \leq \neg \square x \vee \square y$.

Orthomodular complete lattices are examples of Boolean saturated orthomodular lattices. We can embed each orthomodular lattice $\mathcal{L}$ in an element $\mathcal{L}^{\diamond} \in \mathcal{O} \mathcal{M L} \mathcal{L}^{\diamond}$ see (Ref. 9, Theorem 10). In general, $\mathcal{L}^{\diamond}$ is referred as a modal extension of $\mathcal{L}$. In this case we may see the lattice $\mathcal{L}$ as a subset of $\mathcal{L}^{\diamond}$.

Let $\mathcal{H}$ be a complex Hilbert space associated with a quantum system. Since $\mathcal{L}(\mathcal{H})$ is a complete lattice, it is a modal extension of itself. In what follows we build a basic framework for nontrivial modal extensions of Hilbert lattices associated with quantum systems.

For each $r \in \mathcal{L}(\mathcal{H})$, consider the constant symbol $\bar{r}$. We denote by $C_{\mathcal{H}}$ the set of these constants. Let $\left(p_{i}\right)_{i \in I}$ be another family of new constants such that $C_{\mathcal{H}} \cap\left(p_{i}\right)_{i \in I}=\emptyset$. Consider the expansion of the language of $\mathcal{O M} \mathcal{L}^{\diamond}$ obtained by adding the set of constants $C_{\mathcal{H}} \cup\left(p_{i}\right)_{i \in I}$. In this language we want to build a Boolean saturated orthomodular structure, such that it contains the equational theory of $\mathcal{L}(\mathcal{H})$ and that it is able to represent classical consequences through a fix representation of a chosen constant $p_{i}$. Consider the following sets of equations:

- book-keeping axioms: $t\left[\overline{r_{1}}, \ldots, \overline{r_{n}}\right]=\overline{t^{\mathcal{L}(\mathcal{H})}\left[r_{1}, \ldots, r_{n}\right]}$, where $t \in \operatorname{Term}_{\mathcal{O M L}}$ and $r_{1}, \ldots, r_{n}$ $\in \mathcal{L}(\mathcal{H})$

- center axioms: $\left(x, y, p_{i}\right)$ T for each $i \in I$. 
We denote by $E Q(\mathcal{H})$ be the set of book-keeping axioms and by $\mathcal{B}\left(p_{i}\right)$ be the set of center axioms. Let us consider the following equational theory:

$$
\mathcal{O} \mathcal{M L}^{\diamond} \cup E Q(\mathcal{H}) \cup \mathcal{B}\left(p_{i}\right)
$$

If we take the variety $\mathcal{V}_{\mathcal{H}}^{\diamond}$ given by the mentioned equational system, each algebra of $\mathcal{V}_{\mathcal{H}}^{\diamond}$ is a Boolean saturated orthomodular lattice. The set of equations $E Q(\mathcal{H})$ guarantees that the equational theory of $\mathcal{L}(\mathcal{H})$ is maintained in each algebra through the subalgebra $C_{\mathcal{H}}$, which results to be an isomorphic copy of $\mathcal{L}(\mathcal{H})$. The set of equations $\mathcal{B}\left(p_{i}\right)$ guarantees that each $p_{i}$ is a central element in each algebra of $\mathcal{V}_{\mathcal{H}}$. We will see that $\mathcal{V}_{\mathcal{H}}$ is a nonempty class of algebras. Consider the direct product $\Pi_{I} \mathcal{L}(\mathcal{H})$. Since $\Pi_{I} \mathcal{L}(\mathcal{H})$ is a complete orthomodular lattice then it belongs to $\mathcal{O} \mathcal{M} \mathcal{L}^{\diamond}$. The canonical embedding $\mathcal{L}(\mathcal{H}) \hookrightarrow \Pi_{\mathcal{I}} \mathcal{L}(\mathcal{H})$ provides the natural interpretation of the set of constants $C_{\mathcal{H}}$ trivially satisfying the book-keeping axioms. To interpret the constant system $\left(p_{i}\right)_{i \in I}$ is a little more delicate. By Ref. 13, Theorem 11, we have that $Z\left(\Pi_{I} \mathcal{L}(\mathcal{H})\right)=\Pi_{\mathcal{I}} \mathbf{2}$ and then $\operatorname{Card}(I)$ $\leq \operatorname{Card}\left(Z\left(\Pi_{I} \mathcal{L}(\mathcal{H})\right)\right)$. Consequently we can establish an injective function $p: I \hookrightarrow Z\left(\Pi_{I} \mathcal{L}(\mathcal{H})\right)$. Thus we can interpret each constant $p_{i}$ by the element $p(i) \in Z\left(\Pi_{I} \mathcal{L}(\mathcal{H})\right)$, trivially satisfying the center axioms. Hence $\Pi_{I} \mathcal{L}(\mathcal{H})$ is an example of $\mathcal{V}_{\mathcal{H}}^{\diamond}$-algebra and this class is nonempty. Note that for each $A \in \mathcal{V}_{\mathcal{H}}^{\diamond}$, the assignment $r \rightarrow \bar{r}$ gives an orthomodular embedding from $\mathcal{L}(\mathcal{H})$ onto $A$, i.e., $A$ results a nontrivial modal extension of $\mathcal{L}(\mathcal{H})$.

$\mathcal{V}_{\mathcal{H}}$ constitutes a basic framework for nontrivial modal extensions of Hilbert lattices $\mathcal{L}(\mathcal{H})$ in the sense that it provides an underline structure for an algebraic study of the classical consequences of propositions represented in $\mathcal{L}(\mathcal{H})$. In fact, if $p \in \mathcal{L}(\mathcal{H})$ represents a property about the system, any classical consequence of this property will be represented via a constant $p_{i}$. Depending on the kind of classical consequences of the properties of the physical system represented in $\mathcal{H}$ that we want to consider, we would have to eventually add axioms which relate the set $\left(p_{i}\right)_{i \in I}$ and the set $C_{\mathcal{H}}$, giving rise to substructures of $\mathcal{V}_{\mathcal{H}}^{\diamond}$.

Definition 3.2: Let $\mathcal{L}$ be an orthomodular lattice and $\mathcal{L}^{\diamond} \in \mathcal{O} \mathcal{M} \mathcal{L}^{\diamond}$ be a modal extension of $\mathcal{L}$. We define the possibility space of $\mathcal{L}$ in $\mathcal{L}^{\diamond}$ as

$$
\diamond \mathcal{L}=\langle\{\diamond p: p \in \mathcal{L}\}\rangle_{\mathcal{L}^{\diamond}}
$$

The possibility space represents the modal content added to the discourse about properties of the system.

Proposition 3.3: (Reference 9, Proposition 14) Let $\mathcal{L}$ be an orthomodular lattice, $W$ a Boolean sublattice of $\mathcal{L}$, and $\mathcal{L}^{\diamond} \in \mathcal{O} \mathcal{M} \mathcal{L}^{\diamond}$ a modal extension of $\mathcal{L}$. Then $\langle W \cup \diamond \mathcal{L}\rangle_{\mathcal{L}} \diamond$ is a Boolean sublattice of $\mathcal{L}^{\diamond}$. In particular, $\diamond \mathcal{L}$ is a Boolean sublattice of $Z\left(\mathcal{L}^{\diamond}\right)$.

Now, we develop the algebraic counterpart of the classical notion of consequence which will be useful when formalizing the concept of possibility in MWI. As will become clear below, Proposition 3.3 allows to establish a deep relation between this concept and the possibility space.

Definition 3.4: Let $\mathcal{L}$ be an orthomodular lattice, $p \in \mathcal{L}$, and $\mathcal{L}^{\diamond} \in \mathcal{O} \mathcal{M} \mathcal{L}^{\diamond}$ a modal extension of $\mathcal{L}$. Then $x \in \diamond \mathcal{L}$ is said to be a classical consequence of $p$ if and only if for each Boolean sublattice $W$ in $\mathcal{L}$ (with $p \in W$ ) and each Boolean valuation $v: W \rightarrow \mathbf{2}, v(x)=1$ whenever $v(p)=1$. We denote by $\operatorname{Cons}_{\mathcal{L}} \diamond(p)$ the set of classical consequences of $\mathcal{L}$.

Proposition 3.5: Let $\mathcal{L}$ be an orthomodular lattice, $p \in \mathcal{L}$, and $\mathcal{L}^{\diamond} \in \mathcal{O} \mathcal{M} \mathcal{L}^{\diamond}$ a modal extension of $\mathcal{L}$. Then we have that

$$
\operatorname{Cons}_{\mathcal{L}} \diamond(p)=\{x \in \diamond \mathcal{L}: p \leq x\}=\{x \in \diamond \mathcal{L}: \diamond p \leq x\} .
$$

Proof: By definition of $\diamond$ it is clear that $\{x \in \diamond \mathcal{L}: p \leq x\}=\{x \in \diamond \mathcal{L}: \diamond p \leq x\}$ and the inclusion $\{x \in \diamond \mathcal{L}: \diamond p \leq x\} \subseteq \operatorname{Cons}_{\mathcal{L}} \diamond(p)$ is trivial. Let $x \in \operatorname{Cons}_{\mathcal{L}} \diamond(p)$. Assume that $p \not \leq x$. Consider the Boolean subalgebra of $\mathcal{L}$ given by $W=\{p, \neg p, 0,1\}$. By Proposition 3.3, $W^{\diamond}=\langle W \cup \diamond \mathcal{L}\rangle_{\mathcal{L}} \diamond$ is a Boolean sublattice of $\mathcal{L}^{\diamond}$. By the maximal filter theorem, there exists a maximal filter $F$ in $W^{\diamond}$, such that $p \in F$ and $x \notin F$. If we consider the quotient Boolean algebra $W^{\diamond} / F$ and the natural Boolean homomorphism $f: W^{\diamond} \rightarrow W^{\diamond} / F=\mathbf{2}$, then $f(p)=1$ and $f(x)=0$, which is a contradiction. 
Let $\mathcal{L}$ be an orthomodular lattice, $\left(W_{i}\right)_{i \in I}$ the family of Boolean sublattices of $\mathcal{L}$, and $\mathcal{L}^{\diamond}$ a modal extension of $\mathcal{L}$. If $f: \diamond \mathcal{L} \rightarrow \mathbf{2}$ is a Boolean homomorphism, an actualization compatible with $f$ is a global valuation $\left(v_{i}: W_{i} \rightarrow \mathbf{2}\right)_{i \in I}$, such that $v_{i}\left|W_{i} \cap \diamond \mathcal{L}=f\right| W_{i} \cap \diamond \mathcal{L}$ for each $i \in I$. Compatible actualizations represent the passage from possibility to actuality.

Theorem 3.6: (Reference 9, Theorem 19) Let $\mathcal{L}$ be an orthomodular lattice. Then $\mathcal{L}$ admits a global valuation if and only if for each possibility space there exists a Boolean homomorphism $f: \diamond \mathcal{L} \rightarrow \mathbf{2}$ that admits a compatible actualization.

The addition of modalities to the discourse about the properties of a quantum system enlarges its expressive power. At first sight it may be thought that this could help to circumvent contextuality, allowing to refer to physical properties belonging to the system in an objective way that resembles the classical picture. Since the possibility space is a Boolean algebra, there exists a Boolean valuation of the possible properties. But in view of the last theorem, a global actualization that would correspond to a family of compatible valuations is prohibited. Thus, the theorem states that the contextual character is maintained even when the discourse is enriched with modalities.

\section{AN ALGEBRAIC FRAME FOR MANY WORLDS}

In the MWI, all possibilities encoded in the wave function take place, but in different worlds. More precisely, let $\mathcal{M}$ be a physical magnitude represented by an operator $\mathbf{M}$ with spectral decomposition $\mathbf{M}=\sum_{i} a_{i} \mathbf{P}_{i}$. If a measurement of $\mathbf{M}$ is performed and $a_{1}$ occurs, then in another world $a_{2}$ occurs, in some other world $a_{3}$ occurs, etc. Let us now see how we can introduce our modal algebraic frame for MWI.

Let $\mathcal{H}$ be a Hilbert space and suppose that $\mathbf{M}$ has associated a Boolean sublattice $W_{\mathbf{M}}$ of $\mathcal{L}(\mathcal{H})$. The family $\left(\mathbf{P}_{i}\right)_{i}$ is identified as elements of $W_{\mathbf{M}}$. If a measurement is performed and its result is $a_{i}$, this means that we can establish a Boolean homomorphism,

$$
v_{i}: W_{\mathbf{M}} \rightarrow \mathbf{2} \text { s.t. } v_{i}\left(\mathbf{P}_{i}\right)=1 .
$$

\section{A. $\mathcal{O M} \mathcal{L}^{\diamond}$-consequences}

In a possible world where $v_{i}\left(\mathbf{P}_{i}\right)=1$ we will have classical consequences. We can take an arbitrary modal extension $\mathcal{L}^{\diamond}$ of $\mathcal{L}(\mathcal{H})$ and consider the set $\operatorname{Cons}_{\mathcal{L}} \diamond\left(\mathbf{P}_{i}\right)$. The modal extension does not depend on the valuation over the family $\left(\mathbf{P}_{i}\right)_{i}$. Thus, it is clear that the modal extension is independent of any possible world. Modal extensions are simple algebraic extensions of an orthomodular structure. By Proposition 3.5 we have that $\operatorname{Cons}_{\mathcal{L}} \diamond\left(\mathbf{P}_{i}\right)=\left\{x \in \diamond \mathcal{L}(\mathcal{H}): \diamond \mathbf{P}_{i} \leq x\right\}$. Thus, for any arbitrary modal extension $\mathcal{L}^{\diamond}$ of $\mathcal{L}(\mathcal{H})$ in terms of classical consequences, the classical consequences of $v_{i}\left(\mathbf{P}_{i}\right)=1$ are exactly the same ones as $\diamond \mathbf{P}_{i}$ (independently of any possible splitting). In terms of classical consequences which refer to a property $\mathbf{P}_{i}$, it is the same to consider the classical consequences in the possible world where $v_{i}\left(\mathbf{P}_{i}\right)=1$, than to study the classical consequences of $\diamond \mathbf{P}_{i}$ before the splitting.

MWI maintains that in each respective $i$-world, $v_{i}\left(\mathbf{P}_{i}\right)=1$ for each $i$. Thus, a family of valuations $\left(v_{i}\left(\mathbf{P}_{i}\right)=1\right)_{i}$ may be simultaneously considered, each member being realized in each different $i$-world. From an algebraic perspective, this would be equivalent to have a family of pairs $\left\langle\mathcal{L}(\mathcal{H}), v_{i}\left(\mathbf{P}_{i}\right)=1\right\rangle_{i}$, each pair being the orthomodular structure $\mathcal{L}(\mathcal{H})$ with a distinguished Boolean valuation $v_{i}$ over a spectral subalgebra containing $\mathbf{P}_{i}$, such that $v_{i}\left(\mathbf{P}_{i}\right)=1$. In what follows, we will show that the $\mathcal{O} \mathcal{M L}^{\diamond}$ structure is able to capture this fact in terms of classical consequences. For this purpose, the following proposition is needed.

Proposition 4.1: Let $\mathcal{H}$ be Hilbert space, such that $\operatorname{dim}(\mathcal{H})>2$ and $a, b$ be a two distinct atoms in $\mathcal{L}(\mathcal{H})$. If we consider a modal extension $\mathcal{L}^{\diamond}$ of $\mathcal{L}(\mathcal{H})$, then $\diamond(a)=\diamond(b)$.

Proof: Let $a, b$ be two distinct atoms in $\mathcal{L}(\mathcal{H})$. By Ref. 16, Theorem $8(\mathrm{CH} .3)$, there exists a common complement $c$ of $a, b$, i.e., $0=a \wedge c=b \wedge c$ and $1=a \vee c=b \vee c$. Since $\mathcal{L}(\mathcal{H}) \hookrightarrow \mathcal{L}^{\diamond}$ is an $\mathcal{O M L}$-embedding, $c$ is a common complement of $a, b$ in $\mathcal{L}^{\diamond}$. We first note $\neg \diamond \neg c=\square c \leq c$, then $a \wedge \neg \diamond \neg c \leq a \wedge c=0$. Since $\neg \diamond \neg c$ is a central element, $a \leq \diamond \neg c$ and $\diamond a \leq \diamond \neg c$. Since $a \vee c$ $=1$ then $\neg a \wedge \neg c=0$. Therefore, $\neg c \wedge \neg \diamond a \leq \neg a \wedge \neg c=0$. Since $\neg \diamond a$ is a central element then 
$\neg c \leq \diamond a$ and $\diamond \neg c \leq \diamond a$. With the same argument we can prove that $\diamond(b)=\diamond(\neg c)$. Hence $\diamond(a)=\diamond(b)$.

The following theorem is crucial in order to relate MWI with modality in terms of valuations and classical consequences.

Theorem 4.2: Let $\mathcal{H}$ be Hilbert space such that $\operatorname{dim}(\mathcal{H})>2$ and $\left(p_{i}\right)_{i \in I}$ be a family of elements of $\mathcal{L}(\mathcal{H})$, such that $p_{i} \neq 0$ for each $i \in I$. If we consider a modal extension $\mathcal{L}(\mathcal{H}) \hookrightarrow \mathcal{L}^{\diamond}$ then there exists a Boolean homomorphism $v: \diamond \mathcal{L} \rightarrow \mathbf{2}$, such that $v\left(\diamond p_{i}\right)=1$ for each $i \in I$.

Proof: Since $\mathcal{L}(\mathcal{H})$ is an atomic lattice, for each $p_{i}$ there exists an atom $a_{i}$, such that $a_{i} \leq p_{i}$. Let $I_{0}$ be a finite subfamily of $I$. By Proposition 4.1, we have that $0<\wedge_{i \in I_{0}} \diamond\left(a_{i}\right) \leq \wedge_{i \in I_{0}} \diamond\left(p_{i}\right)$. Therefore, the family $\left(\diamond p_{i}\right)_{1 \in I}$ generates a proper filter $F$ in the Boolean algebra $\diamond \mathcal{L}$. Extending $F$ to a maximal filter $F_{\mathbf{M}}$, the natural Boolean homomorphism $v: \diamond \mathcal{L} \rightarrow \mathbf{2}$ satisfies that for each $i \in I, v\left(\diamond p_{i}\right)=1$.

While MWI considers a family of pairs $\left\langle\mathcal{L}(\mathcal{H}), v_{i}\left(\mathbf{P}_{i}\right)=1\right\rangle_{i}$ for each possible $i$-world and the classical consequences of $v_{i}\left(\mathbf{P}_{i}\right)=1$ in the $i$-world, the $\mathcal{O} \mathcal{M} \mathcal{L}^{\diamond}$ structure, by Proposition 3.5, considers classical consequences of each $v_{i}\left(\mathbf{P}_{i}\right)=1$ coexisting simultaneously in one and the same structure, what is possible in view of Theorem 4.2. More precisely, as a valuation $v: \diamond \mathcal{L} \rightarrow \mathbf{2}$ exists such that $v\left(\diamond \mathbf{P}_{i}\right)=1$ for each $i$, each element $x \in \diamond \mathcal{L}$ such that $\mathbf{P}_{i} \leq x$ necessarily satisfies $v(x)=1$.

\section{B. Many worlds and Kochen-Specker-type theorems}

KS theorem does not impose conditions on both the family of valuations $v_{i}\left(\mathbf{P}_{i}\right)=1$, considered as a family of pairs $\left\langle\mathcal{L}(\mathcal{H}), v_{i}\left(\mathbf{P}_{i}\right)=1\right\rangle_{i}$ in MWI nor on the Boolean valuation $v: \diamond \mathcal{L}(\mathcal{H}) \rightarrow \mathbf{2}$ satisfying $v\left(\diamond \mathbf{P}_{i}\right)=1$ for each $i$ in the $\mathcal{O} \mathcal{M} \mathcal{L}^{\diamond}$ structure (Theorem 4.2). In fact, by Theorem 3.6 KS only prevents from extending the valuation $v: \diamond \mathcal{L}(\mathcal{H}) \rightarrow \mathbf{2}$ to $\mathcal{L}(\mathcal{H})$ in a compatible manner. In the wording previous to Theorem 3.6, KS theorem prohibits to pass from the realm of possibility to that of actuality in the sense that it precludes to establish a compatible actualization for $v: \diamond \mathcal{L}(\mathcal{H}) \rightarrow \mathbf{2}$ to $\mathcal{L}(\mathcal{H})$ when $\operatorname{dim}(\mathcal{H})>2$ (see Theorem 3.1).

In its algebraic version given in Theorem 3.1, KS only imposes a limit to the possibility of establishing compatible valuations over $\mathcal{L}(\mathcal{H})$ when $\operatorname{dim}(\mathcal{H})>2$ but does not cause incompatibilities when reference is made to possible global valuations in the realm of possibilities considered in the $\mathcal{O} \mathcal{M L}^{\diamond}$ structure or in the family $\left\langle\mathcal{L}(\mathcal{H}), v_{i}\left(\mathbf{P}_{i}\right)=1\right\rangle_{i}$ from MWI. Thus, valuations over different $i$-worlds are admitted.

\section{CONCLUSIONS}

In this paper we have analyzed the orthomodular formal structure of quantum mechanics in relation to both MWI and MI. In order to deal with logical possibility in these interpretations, we considered two different algebraic approaches which were characterized in Secs. III and IV. In the case of MWI, the structure is the family of pairs $\left\langle\mathcal{L}(\mathcal{H}), v_{i}\left(P_{i}\right)=1\right\rangle_{i}$ of orthomodular lattices with a distinguished Boolean valuation that assigns "true" to a projector of a spectral algebra in each one of them. For MI, we have the Boolean saturated orthomodular lattice. Both structures allow us to compare the role of contextuality in relation to the formal account of actual and possible properties in a rigorous way as it is shown in Table I.

The modal scheme we developed in Ref. 9, i.e., the Boolean saturated orthomodular lattice, is also adequate to consider the notion of possibility within MWI. The whole set of possible worlds, each one with an actualized value of a property, is algebraically equivalent to the set of valuations to "true" of the possible properties in $\mathcal{L} \diamond$. That is to say, the actualization of each value of a given property in each $i$-world is analogous to the assignment of the value "true" to all possible properties in the scheme of MI.

We have shown that the KS theorem only imposes a limit to the possibility of establishing compatible valuations over $\mathcal{L}(\mathcal{H})$. However, there is no incompatibility when reference is only made to valuations in the realm of possibilities, i.e., in the $\mathcal{O} \mathcal{M L} \mathcal{L}^{\diamond}$ structure for MI or in the family $\left\langle\mathcal{L}(\mathcal{H}), v_{i}\left(\mathbf{P}_{i}\right)=1\right\rangle_{i}$ for MWI. 
TABLE I. Modality and MWI under $\mathcal{O M L}^{\diamond}$-structure.

\begin{tabular}{|c|c|c|}
\hline & Modality & MWI \\
\hline Valuations & $\begin{array}{l}\text { There exists a Boolean valuation } \\
v: \diamond(\mathcal{L}(\mathcal{H})) \rightarrow \mathbf{2} \text { such } \\
\text { that } v\left(\diamond P_{i}\right)=1 \text { for each } i\end{array}$ & $\begin{array}{c}\text { Families of Boolean valuations } \\
\left(v_{i}\left(P_{i}\right)=1\right)_{i}, \text { may be simultaneously } \\
\text { considered, each member } \\
\text { being realized in each } \\
\text { different } i \text {-world }\end{array}$ \\
\hline KS theorem & $\begin{array}{l}\text { Precludes to establish } \\
\text { compatible actualizations for } \\
v: \diamond(\mathcal{L}(\mathcal{H})) \rightarrow \mathbf{2} \text { to } \mathcal{L}(\mathcal{H})\end{array}$ & $\begin{array}{l}\text { Does not cause incompatibility } \\
\text { when each member of a family } \\
\text { of valuations }\left(v_{i}\left(P_{i}\right)=1\right)_{i} \text { is } \\
\text { considered }\end{array}$ \\
\hline
\end{tabular}

\section{ACKNOWLEDGMENTS}

This work was partially supported by the following grants: Grant No. PICT 04-17687 (ANPCyT), PIP Grant No. 6461/05 (CONICET), UBACyT Grant Nos. X081 and X204, and Projects of the Fund for Scientific Research Flanders G.0362.03 and G.0452.04. G. D. and H. F. are Fellows of the Consejo Nacional de Investigaciones Científicas y Técnicas (CONICET).

${ }^{1}$ Bacciagaluppi, G., “A Kochen Specker theorem in the modal interpretation of quantum mechanics,” Int. J. Theor. Phys. 34, 1205 (1995).

${ }^{2}$ Burris, S. and Sankappanavar, H. P., A Course in Universal Algebra, Graduate Text in Mathematics (Springer-Verlag, New York, 1981).

${ }^{3}$ Birkhoff, G. and von Neumann, J., “The logic of quantum mechanics," Ann. Math. 37, 823 (1936).

${ }^{4}$ Dieks, D., "Probability in the modal interpretations of quantum mechanics," Stud. Hist. Philos. Mod. Phys. 38, 292 (2007).

${ }^{5}$ Dieks, D., "The formalism of quantum theory: an objective description of reality," Ann. Phys. 500, 174 (1988).

${ }^{6}$ Deutsch, D., "Quantum theory as a universal physical theory," Int. J. Theor. Phys. 24, 1 (1985).

${ }^{7}$ DeWitt, B. S. and Graham, N., The Many-Worlds Interpretation of Quantum Mechanics (Princeton University Press, Princeton, 1973).

${ }^{8}$ Domenech, G. and Freytes, H., "Contextual logic for quantum systems," J. Math. Phys. 46, 012102 (2005).

${ }^{9}$ Domenech, G., Freytes, H., and de Ronde, C., "Scopes and limits of modality in quantum mechanics," Ann. Phys. 15, 853 (2006).

${ }^{10}$ Domenech, G., Freytes, H., and de Ronde, C., "A topological study of contextuality and modality in quantum mechanics," Int. J. Theor. Phys. 47, 168 (2008).

${ }^{11}$ Everett, H., “"Relative state' formulation of quantum mechanics," Rev. Mod. Phys. 29, 454 (1957).

${ }^{12}$ Hemmo, M., "Quantum mechanics without collapse: Modal interpretations, histories and many worlds," Ph.D. dissertation, University of Cambridge, 1996.

${ }^{13}$ Iskander, A., "Factorable congruences and strict refinement," Acta Math. Univ. Comen. 65, 101 (1996).

${ }^{14}$ Jauch, J. M., Foundations of Quantum Mechanics (Addison-Wesley, Reading, MA, 1968).

${ }^{15}$ Kalman, J. A., "Lattices with involution," Trans. Am. Math. Soc. 87, 485 (1958).

${ }^{16}$ Kalmbach, G., Ortomodular Lattices (Academic, London, 1983).

${ }^{17}$ Kochen, S. and Specker, E., "On the problem of hidden variables in quantum mechanics," J. Math. Mech. 17, 59 (1967).

${ }^{18}$ Kochen, S., in Symposium on the Foundations of Modern Physics, 1985, edited by Lathi, P. and Mittelslaedt, P. (World Scientific, Johensuu, 1985), pp. 151-169.

${ }^{19}$ Maeda, F. and Maeda, S., Theory of Symmetric Lattices (Springer-Verlag, Berlin, 1970).

${ }^{20}$ Wheeler, J. A., "Assessment of Everett's 'relative state' formulation of quantum mechanics," Rev. Mod. Phys. 29, 463 (1957).

${ }^{21}$ van Fraassen, B. C., in Contemporary Research in the Foundations and Philosophy of Quantum Theory, edited by Hooker, C. A. (Reidel, Dordrecht, 1973). 